\title{
An inverse problem in the economic theory of demand
}

\section{Problème inverse sur la théorie économique de la demande}

\author{
Ivar Ekeland ${ }^{\mathrm{a}}$, Ngalla Djitté ${ }^{\mathrm{b}, *}$ \\ a Canada Research Chair in Mathematical Economics, University of British Columbia, Canada \\ ${ }^{\mathrm{b}}$ Laboratoire d'Analyse Numérique et d'Informatique, Université Gaston Berger de Saint-Louis, Sénégal
}

Received 10 February 2005; received in revised form 12 October 2005; accepted 17 October 2005

Available online 2 December 2005

\begin{abstract}
Given an exchange economy consisting of $k$ consumers, there is an associated collective demand function, which is the sum of the individual demand functions. It maps the price system $p$ to a goods bundle $x(p)$. Conversely, given a map $p \rightarrow x(p)$, it is natural to ask whether it is the collective demand function of a market economy. We answer that question in the case when $k$ is less than the number of goods $n$. The proof relies on finding convex solutions to a strongly nonlinear system of partial differential equations.
\end{abstract}

๑) 2006 L'Association Publications de l'Institut Henri Poincaré. Published by Elsevier B.V. All rights reserved

\section{Résumé}

La fonction de demande agrégée d'une société composée de $k$ individus résulte de la sommation de $k$ fonctions de demandes individuelles. Elle fait correspondre à un système de prix $p$, un vecteur de biens $x(p)$. Inversement, étant donnée une fonction $p \rightarrow x(p)$, est-elle une fonction de demande agrégée ? Nous apporterons une réponse à cette question dans le cas où il y a moins de consommateurs que de biens.

C 2006 L'Association Publications de l'Institut Henri Poincaré. Published by Elsevier B.V. All rights reserved

Keywords: Utility function; Aggregate demand function; Inverses problems; Exteriors differentials systems; Integrals manifolds

Mots-clés : Fonction d'utilité ; Demande agrégée; Problèmes inverses ; Systèmes différentiels extérieurs ; Éléments intégraux ; Variétés intégralles

\section{The disaggregation problem}

Consider an exchange economy consisting of $k$ consumers. The space of goods is $\mathbb{R}^{n}$, and agent $i$ is characterized by a strictly quasi-concave utility function $u_{i}$ and a wealth $c_{i}$. Given a set of non-negative prices $p \in \mathbb{R}_{+}^{n}$, agent $i$ chooses its consumption $x_{i}(p)$ by solving the optimization problem:

\footnotetext{
* Corresponding author.

E-mail address: ngalla@ ceremade.dauphine.fr (N. Djitté).
} 


$$
\begin{aligned}
& \max _{x} u_{i}(x), \\
& p^{\prime} x \leqslant c_{i} .
\end{aligned}
$$

Assume that, for every $p$ belonging to a convex subset $\Omega$ of $\mathbb{R}^{n}$, the solution $x_{i}(p)$ exists, and satisfies $p^{\prime} x_{i}(p)=c_{i}$. This will be the case, for instance, if the $u_{i}$ are increasing with respect to every argument, and we can take $\Omega=\mathbb{R}^{n}$. The individual demands $x_{i}(p)$ are then well-defined on $\Omega$, and adding them up, we obtain the market demand:

$$
x(p):=\sum_{i=1}^{k} x_{i}(p) .
$$

It obviously satisfies the Walras law:

$$
p^{\prime} x(p)=\sum_{i=1}^{k} c_{i}=c .
$$

We are interested in the inverse problem: given a map $p \rightarrow x(p)$ from a subset $\Omega \subset \mathbb{R}^{n}$ into $\mathbb{R}^{n}$, an integer $k$, and numbers $w_{i}, 1 \leqslant i \leqslant k$, can one find strictly concave utility functions $u_{i}(x), 1 \leqslant i \leqslant k$, such that the decomposition (3) holds, $x_{i}(p)$ being the solution of problem (1)? An obvious necessary condition is that $x(p)$ should satisfy the Walras law, but is it sufficient?

This is the famous disaggregation problem, which has a long history. In the case when $k \geqslant n$ (there are more consumers than goods), it was proved by Chiappori and Ekeland in [4] that the Walras law is sufficient. Much earlier, in a celebrated series of papers, Sonnenschein, Mantel and Debreu had investigated the same question in the case of excess demand, that is when the linear constraint is of the form $p^{\prime} x \leqslant p^{\prime} \omega_{i}$, where $\omega_{i} \in \mathbb{R}^{n}$ is the initial endowment of agent $i$. They proved that if $k \geqslant n$, the Walras law was sufficient. We refer to [13] for a history of the SonnenscheinDebreu-Mantel results, and to [5] for a recent proof, in the spirit of the present paper.

Much less is known in the case when $k<n$, except in the case $k=1$, where the group reduces to one person, so that collective demand coincides with individual demand. It is the aim of the present paper to fill that gap in the case of market demand. We do not treat the case of excess demand, which, to our knowledge, still remains open.

The case $k=1$ is classical. It was first treated by Antonelli [1], whose results went forgotten and were rediscovered by Slutsky [14]. We summarize these results, and sketch part of the proof; the reader will find the full argument in [12], for instance.

Here and later, we denote differentiation by the symbol $D$, so that $D u$ denotes the Jacobian matrix of the map $u$ :

$$
D u=\left(\frac{\partial u^{i}}{\partial p_{j}}\right)_{1 \leqslant j \leqslant n}^{1 \leqslant i \leqslant n}
$$

and $D^{2} u$ denotes the matrix of second derivatives of the function $u$ :

$$
D^{2} u=\left(\frac{\partial^{2} u}{\partial p_{j} \partial p_{j}}\right)_{1 \leqslant j \leqslant n}^{1 \leqslant i \leqslant n}
$$

Proposition 1. Let $\Omega$ be a convex open subset of $\mathbb{R}^{n}$, and $x(p)$ be a $C^{1}$ map from $\Omega$ into $\mathbb{R}^{n}$ satisfying the Walras law. A necessary and sufficient condition for $x(p)$ to be the individual demand associated with some quasi-concave utility function $u$ is that the restriction of $D x(p)$ to $[x(p)]^{\perp}$ is symmetric and negative definite for every $p \in \Omega$.

In other words, we can write:

$$
D x=Q+\xi x^{\prime}
$$

where $Q$ is a symmetric,negative definite matrix, and $\xi$ is a vector (so that the last term in the above equation is a matrix of rank 1). This is known in the literature as the Slutsky condition. For an analogue of this condition in the case of individual excess demand, we refer to [5].

Let us sketch the proof that the Slutsky condition is necessary. Assume that the utilitity function $u$ of the sole agent is $C^{2}$, and that the restriction of $D^{2} u(x(p))$ to $[p]^{\perp}$ is negative definite. Then the individual demand $x(p)$ is characterized by the first-order optimality condition: 


$$
\begin{aligned}
& D u(x(p))=\lambda(p) p, \\
& p^{\prime} x(p)=c
\end{aligned}
$$

where $\lambda(p)>0$ is the Lagrange multiplier. Applying the Inverse Function Theorem to this system, one finds that the map $x(p)$ and the function $\lambda(p)$ are well-defined and $C^{1}$ on $\Omega$.

Introduce now the indirect demand function:

$$
v(p)=\max _{x}\left\{u(x) \mid p^{\prime} x \leqslant c\right\} .
$$

The function $v(p)$ is quasi-convex and $C^{2}$. More precisely, the restriction of $D^{2} v(p)$ to $[x(p)]^{\perp}$ is positive definite. In addition, $u(x)$ can be obtained from $v(p)$ by the formula:

$$
u(x)=\min _{p}\left\{v(p) \mid p^{\prime} x \geqslant c\right\} .
$$

Let us differentiate $v(p)$ by applying the envelope theorem to the formula $v(p)=\max _{x}\left\{u(x)-\lambda(p)\left(c-p^{\prime} x\right)\right\}$. We get:

$$
\begin{aligned}
& D v(p)=-\lambda(p) x(p), \\
& x(p)=-\mu(p) D v(p)
\end{aligned}
$$

where $\mu(p)=1 / \lambda(p)$. Differentiating, we get:

$$
D x=-\mu D^{2} v+(D \mu)(D v)^{\prime} .
$$

The last term on the right-hand side vanish on $[x(p)]^{\perp}=[D v(p)]^{\perp}$, so that the restriction of $D x(p)$ to $[x(p)]^{\perp}$ is negative definite, and the Slutsky condition is indeed necessary. The fact that it is also sufficient is proved by using formula (6); we refer to [12] for details.

In the case $k>1$, there is a natural extension of the Slutsky condition, which was first described by Diewert [6] and by Geanakoplos and Polemarchakis [9] in the context of market demand:

Definition 2. Let $\Omega$ be a convex open subset of $\mathbb{R}^{n}$, and $x(p)$ be a $C^{2}$ map from $\Omega$ into $\mathbb{R}^{n}$ satisfying the Walras law. We shall say that $x(p)$ satisfies the generalized Slutsky condition for $k$ consumers, abbrevieated to GS $(k)$, if for every $p \in \Omega$ we have:

$$
D x(p)=Q(p)+\sum_{i=1}^{k} \xi_{i}(p) \eta_{i}(p)^{\prime}
$$

where $Q(p)$ is symmetric and negative definite, and the vectors $\eta_{i}, 1 \leqslant i \leqslant k$, contain $x(p)$ in their linear span.

Proposition 3. Let $\Omega$ be a convex open subset of $\mathbb{R}^{n}$, and $x(p)$ be a $C^{2}$ map from $\Omega$ into $\mathbb{R}^{n}$ satisfying the Walras law. A necessary condition for $x(p)$ to be the market demand for an exchange economy with $k$ consumers is that if $x(p)$ satisfies $\mathrm{GS}(k)$ on $\Omega$.

Proof. Rewrite formula (3), using formula (8) for each individual demand $x_{i}(p)$. We get:

$$
x(p)=-\sum_{i=1}^{k} \mu_{i}(p) D v_{i}(p) .
$$

Now we differentiate:

$$
D x=-\sum_{i=1}^{k} \mu_{i} D^{2} v_{i}-\sum_{i=1}^{k} D \mu_{i}\left(D v_{i}\right)^{\prime} .
$$

The first term on the right is a symmetric matrix, and the second has the desired form $\sum_{i=1}^{k} \xi_{i} \eta_{i}^{\prime}$, with $\eta_{i}=D v_{i}$. Because of relation (10), $x(p)$ belongs to the $k$-dimensional space $E_{k}(p)=\operatorname{Span}\left[\left(D v_{i}(p)\right) \mid 1 \leqslant i \leqslant k\right]$. Finally, 
$E_{k}(p)^{\perp}$ is the intersection of all the $\left[D v_{i}(p)\right]^{\perp}=\left[x_{i}(p)\right]^{\perp}$, and the restriction of $D^{2} v_{i}(p)$ to $\left[x_{i}(p)\right]^{\perp}$ is positive definite because of the Slutsky condition. So the restriction of $D x(p)$ to $E_{k}(p)^{\perp}$ must be negative definite. Writing:

$$
D x=\left(-\sum_{i=1}^{k} \mu_{i} D^{2} v_{i}-\sum_{i=1}^{k} \alpha_{i} D v_{i}\left(D v_{i}\right)^{\prime}\right)-\sum_{i=1}^{k}\left(D \mu_{i}+\alpha_{i} D v_{i}\right)\left(D v_{i}\right)^{\prime}
$$

and taking $\alpha_{i}>0$ large enough, we find that the first term on the right-hand side is symmetric and negative definite, and this is the $\mathrm{GS}(k)$ condition.

This paper aims at showing that the $\mathrm{GS}(k)$ condition is sufficient. We will prove the following:

Theorem 4. Let $x(p)$ be an analytic map from some convex neighbourhood $\Omega_{0}$ of $\bar{p} \in \mathbb{R}^{n}$ into $\mathbb{R}^{n}$ satisfying $p^{\prime} x(p)=$ $w$ for some $w>0$. Assume that $x(p)$ satisfies the $\mathrm{GS}(k)$ condition on $\Omega_{0}$ for some $k>1$. Then, given any family $w_{i}>0,1 \leqslant i \leqslant k$, such that $\sum w_{i}=w$, there exists some convex neighbourhood $\Omega_{1} \subset \Omega_{0}$ of $\bar{p}$, such that $x(p)$ is the market demand function for an exchange economy with $k$ consumers with wealths $w_{1}, \ldots, w_{k}$.

This theorem will be a consequence of another one, which we state in the next section.

\section{A system of nonlinear PDEs}

Let us rephrase the disaggregation problem as a system of partial differential equations. We are given a constant $c$, and a map $x(p)$ satisfying $p^{\prime} x(p)=c>0$. We choose positive constants $c_{1}, \ldots, c_{k}$ which sum up to $c$, and we seek maps $x_{1}(p), \ldots, x_{k}(p)$ such that $p^{\prime} x_{i}(p)=c_{i}$ and $x_{i}(p)$ solves the optimization problem (1), (2) for some strictly quasi-concave function $u^{i}$. Substituting the expression (8) for each $x_{i}(p)$, we get the system of equations:

$$
\begin{aligned}
& x(p)=-\sum_{i=1}^{k} \mu_{i}(p) D v_{i}(p), \\
& p^{\prime} D v_{i}(p)=-\frac{c_{i}}{\mu_{i}(p)}, \quad 1 \leqslant i \leqslant k .
\end{aligned}
$$

Conversely, if there is a set of functions $v_{i}(p)$ and $\mu_{i}(p), 1 \leqslant i \leqslant k$, satisfying this system, if the $v_{i}(p)$ are $C^{2}$ with $D^{2} v_{i}$ positive definite, and if the $\mu_{i}(p)$ are positive, then the $u_{i}(x)$ defined by formula (6) will be $C^{2}$ and strictly quasi-concave, and $x_{i}(p)$ will maximize $u_{i}(x)$ under the constraint $p^{\prime} x \leqslant w_{i}$. So the disaggregation problem is equivalent to finding solutions $\left(v_{i}, \mu_{i}\right), 1 \leqslant i \leqslant k$, of the system (12), (13) with the $v_{i}$ convex and the $\mu_{i}$ positive.

Let us rewrite the system as follows:

$$
\begin{aligned}
& -\sum_{i=1}^{k} \mu_{i} \frac{\partial v_{i}}{\partial p_{j}}=x^{j}(p), \quad 1 \leqslant j \leqslant n, \\
& -\mu_{i} \sum_{j=1}^{n} p_{j} \frac{\partial v_{i}}{\partial p_{j}}=c_{i}, \quad 1 \leqslant i \leqslant k .
\end{aligned}
$$

Theorem 5. Let $x(p)$ be an analytic map from some convex neighbourhood $\Omega_{0}$ of $\bar{p} \in \mathbb{R}^{n}$ into $\mathbb{R}^{n}$ satisfying $p^{\prime} x(p)=$ $\sum_{i=1}^{k} c_{i}$. If $x(p)$ satisfies the $\mathrm{GS}(k)$ condition on $\Omega_{0}$ for some $k>1$, then there exists some neighbourhood $\Omega_{1} \subset \Omega_{0}$ of $\bar{p}$, and analytic functions $v_{i}(p)$ and $\mu_{i}(p), 1 \leqslant i \leqslant k$, defined on $\Omega_{1}$, with $D^{2} v_{i}$ positive definite and $\mu_{i}>0$, satisfying equations (14) and (15).

Of course, we can eliminate the $\mu_{i}$ from the equations. We then get a system of $n$ nonlinear partial differential equations for the $k$ unknown functions $v_{i}$ :

$$
\sum_{i=1}^{k} \frac{c_{i}}{\sum_{j=1}^{n} p_{j} \frac{\partial v_{i}}{\partial p_{j}}} \frac{\partial v_{i}}{\partial p_{j}}=x^{j}(p), \quad 1 \leqslant j \leqslant n .
$$


This can be written in a more transparent way by introducing the vector field:

$$
\pi(p)=\sum_{j=1}^{n} p_{j} \frac{\partial}{\partial p_{j}}
$$

and recognizing in the denominator of (16) the derivative of $v_{i}$ in the direction $\pi$, which we denote by $\partial / \partial \pi$ :

$$
\sum_{i=1}^{k} c_{i} \frac{\partial v_{i} / \partial p_{j}}{\partial v_{i} / \partial \pi}=x^{j}(p), \quad 1 \leqslant j \leqslant n .
$$

Again, the problem consists in finding convex solutions $v_{1}, \ldots, v_{k}$ of system (18), and the answer is provided by Theorem 5: it is possible, provided the $x^{i}(p)$ satisfy the Walras law and the GS $(k)$ condition, and they are analytic.

Before we proceed with the proof, let us comment on related results. If $k<n$, the system is clearly overdetermined (there are more equations than unknown functions), and some compatibility condition on the right-hand sides $x^{j}(p), 1 \leqslant i \leqslant n$, is needed in order to find solutions; this is what Theorem 5 provides, with the added twist that we want the solutions to be convex.

When there is a single equation, $k=1$, the results of the preceding section show that there is a quasi-convex solution $v_{1}(p)=v(p)$ provided the $x^{j}(p)$ satisfy the Walras law and the Slutsky condition. This solution is defined globally (there is no need to restrict the initial neighbourhood), and it does not require that the $x^{j}(p)$ be analytic: $C^{2}$ is enough. On the other hand, it does not give a convex solution, only a quasi-convex one, so the conclusion might seem to be weaker. This is not so; indeed, given a quasi-convex function $v$ and a point $\bar{p}$, we can always find a function $\varphi(t)$ such that $\varphi \circ v$ is convex in some neighbourhood of $\bar{p}$, and we then take advantage of the following remark.

Lemma 6. If $\left(v_{1}, \ldots, v_{k}\right)$ is a solution of system (18) near $\bar{p}$ and the $\varphi_{i}: \mathbb{R} \rightarrow \mathbb{R}$ satisfies $\varphi^{\prime}\left(v_{i}(\bar{p})\right) \neq 0$, then $\left(\varphi_{1} \circ v_{1}, \ldots, \varphi_{k} \circ v_{k}\right)$ is also a solution of system (18) near $\bar{p}$.

Proof. Clear.

When $k \geqslant n$, then the $\mathrm{GS}(k)$ condition is vacuous, and all we are left with is the Walras law. Indeed, it has been proved by Chiappori and Ekeland [4] that in that case, the system (14), (15) always has local solutions $v_{i}$ and $\mu_{i}$, with the $v_{i}$ convex and the $\mu_{i}$ positive, provided only that the $x^{i}(p)$ are analytic and satisfy $\sum p_{j} x^{j}(p)=\sum c_{i}$.

When $k<n$, and we consider only part of the system, that is equations (14) but not the constraints (15), then it has been proved by Ekeland and Nirenberg [8] that there are local solutions $v_{i}$ and $\mu_{i}$, with the $v_{i}$ convex and the $\mu_{i}$ positive, provided the $x^{j}(p)$ are $C^{2}$ (not necessarily analytic) and satisfy the GS $(k)$ condition.

This paper is the first one to consider the full problem with $k<n$. The proof is provided in the next section. It relies, as [4], on the Cartan-Kähler theorem, which is a very sophisticated tool for solving systems of partial differential equations in the analytic framework; see [3] for a detailed statement and proof. Let us conclude by remarking on the requirement that the right-hand sides $x^{j}(p)$ be analytic. This means that they can be expanded in convergent power series in the neighbourhood of any point $p$. This is much stronger than simply being indefinitely differentiable: the Taylor series has to converge. The reason this is needed is that the proof of the Cartan-Kähler theorem goes by expanding both sides of the equations in power series and matching coefficients. Whether Theorem 5 still holds true if the right-hand sides $x^{j}(p)$ are only assumed to be $C^{\infty}$ is not known; we have investigated the question without success.

\section{Solving the system}

\subsection{Some simplifications}

We will give the proof only in the case $k=2$. This will considerably simplify the notations, and make the argument more transparent by getting rid of half the indices. We will also take $c_{1}=c_{2}=1$. Let us restate the problem in that case.

We are given an analytic map $x(p)$ of $\Omega_{0} \subset \mathbb{R}^{n}$ into $\mathbb{R}^{n}$ satisfying the Walras law:

$$
p^{\prime} x(p)=2
$$


and the $\mathrm{GS}(k)$ condition:

$$
D x(p)=Q(p)+\xi_{1}(p) \eta_{1}(p)^{\prime}+\xi_{2}(p) \eta_{2}(p)^{\prime}
$$

where $Q(p)$ is symmetric and negative definite, and $x(p)$ belongs to the 2-dimensional subspace:

$$
E_{2}(p)=\operatorname{Span}\left[\eta_{1}(p) \eta_{2}(p)\right]
$$

We want to find functions $u(p), v(p), \lambda(p)$ and $\mu(p)$ such that $u$ and $v$ are convex, $\lambda$ and $\mu$ are negative, and:

$$
\begin{aligned}
& \lambda \frac{\partial u}{\partial p_{j}}+\mu \frac{\partial v}{\partial p_{j}}=x^{j}(p), \quad 1 \leqslant j \leqslant n, \\
& \lambda \sum_{j=1}^{n} p_{j} \frac{\partial u}{\partial p_{j}}=1 \\
& \mu \sum_{j=1}^{n} p_{j} \frac{\partial v}{\partial p_{j}}=1 .
\end{aligned}
$$

This system naturally splits in two parts: the $n$ Eqs. (21), which express the vector $x(p)$ as a linear combination of the two gradients $D u$ and $D v$ :

$$
\lambda(p) D u+\mu(p) D v=x(p)
$$

and the two Eqs. (22) and (23), which are constraints on the coefficients $\lambda$ and $\mu$ :

$$
\begin{aligned}
& \lambda\left(p^{\prime} D u\right)=1, \\
& \mu\left(p^{\prime} D v\right)=1 .
\end{aligned}
$$

If we define new variables $u^{i}$ and $v^{j}$ by:

$$
\begin{aligned}
u^{i} & :=\frac{\partial u}{\partial p_{i}}, \\
v^{i} & :=\frac{\partial v}{\partial p_{i}}
\end{aligned}
$$

Eqs. (21), (22) and (23) become algebraic relations between the $u^{i}, v^{j}, \lambda$ and $\mu$ :

$$
\begin{aligned}
& \lambda u^{i}+\mu v^{i}=x^{i}(p), \quad 1 \leqslant i \leqslant n, \\
& \lambda \sum_{j=1}^{n} p_{j} u^{j}=1, \\
& \mu \sum_{j=1}^{n} p_{j} v^{j}=1 .
\end{aligned}
$$

Define $\mathcal{M}$ to be the set of all $\left(p_{i}, u^{j}, v^{k}, \lambda, \mu\right), 1 \leqslant i, j, k \leqslant n$, satisfying (27), (28) and (29). The problem consists in finding convex functions $u$ and $v$, negative functions $\lambda$ and $\mu$ such that:

$$
(p, D u(p), D v(p), \lambda(p), \mu(p)) \in \mathcal{M} \quad \forall p .
$$

In other words, we are looking for holonomic sections of $\mathcal{M}$ over $\mathbb{R}^{n}$.

\subsection{Finding general solutions}

In order to study the manifold $\mathcal{M}$ and to have a convenient setting for the Cartan-Kähler theorem we will change coordinates in $\mathbb{R}^{n}$. We will then apply the Cartan-Kähler theorem to find holonomic sections of $\mathcal{M}$ over $\mathbb{R}^{n}$, that is, to find functions $u, v, \lambda$ and $\mu$ which solve the system (21), (22) and (23) but which do not necessarily satisfy the further requirements of convexity and negativity. 
Define a differential 1-form $\omega$ by:

$$
\omega=\sum_{i=1}^{n} x^{i}(p) d p_{i}
$$

Because of the GS( $k$ ) condition (20), we have:

$\omega \wedge \mathrm{d} \omega \wedge \mathrm{d} \omega=0$.

By the Darboux theorem (see [3] or [8]), we can find functions $\left(q_{1}(p), q_{2}(p), q_{3}(p), q_{4}(p)\right)$ such that:

$$
\omega=q_{1} \mathrm{~d} q_{2}+q_{3} \mathrm{~d} q_{4} .
$$

Find other functions $q_{i}(p), 5 \leqslant i \leqslant n$, such that the $D q_{i}(\bar{p}), 1 \leqslant i \leqslant n$, are linearly independent, and use the $q_{i}$ as a nonlinear coordinate system near $\bar{p}$.

Note that, setting $h=q_{1} / q_{3}$, we have:

$$
\omega \wedge \mathrm{d} \omega=\left(q_{3} \mathrm{~d} q_{1}-q_{1} \mathrm{~d} q_{3}\right) \wedge \mathrm{d} q_{2} \wedge \mathrm{d} q_{4}=q_{3}^{2} \mathrm{~d} h \wedge \mathrm{d} q_{2} \wedge \mathrm{d} q_{4} .
$$

If there is another splitting of $\omega$, namely $\omega=\lambda \mathrm{d} u+\mu \mathrm{d} v$, then we must also have:

$$
\omega \wedge \mathrm{d} \omega=(\mu \mathrm{d} \lambda-\lambda \mathrm{d} \mu) \wedge \mathrm{d} u \wedge \mathrm{d} v=\mu^{2} \mathrm{~d}\left(\frac{\lambda}{\mu}\right) \wedge \mathrm{d} u \wedge \mathrm{d} v .
$$

Comparing formulas (34) and (35), we find that $\left(\mathrm{d} h, \mathrm{~d} q_{2}, \mathrm{~d} q_{4}\right)$ and $\left(\mathrm{d}\left(\frac{\lambda}{\mu}\right), \mathrm{d} u, \mathrm{~d} v\right)$ span the same subspace. It follows that $\mathrm{d} u$ and $\mathrm{d} v$ must belong to the 3 -dimensional subspace $E_{3}(p)=\operatorname{Span}\left[\mathrm{d} q_{2}, \mathrm{~d} q_{4}, \mathrm{~d} h\right]$. Writing:

$$
\mathrm{d} u=\sum_{i=1}^{n} u^{i} \mathrm{~d} q_{i}
$$

we find that in fact:

$$
\mathrm{d} u=u^{2} \mathrm{~d} q_{2}+u^{4} \mathrm{~d} q_{4}+u^{h} \mathrm{~d} h=u^{2} \mathrm{~d} q_{2}+u^{4} \mathrm{~d} q_{4}+\frac{1}{q_{3}} u^{h} \mathrm{~d} q_{1}-\frac{q_{1}}{q_{3}^{2}} u^{h} \mathrm{~d} q_{3} .
$$

Comparing formulas (36) and (37), we find that:

$$
\begin{aligned}
& q_{1} u^{1}+q_{3} u^{3}=0, \\
& u^{i}=0, \quad 5 \leqslant i \leqslant n
\end{aligned}
$$

and similar relations for the $v^{j}$.

In this coordinate system, $\mathcal{M}$ is the set of $\left(q_{i}, u^{j}, v^{k}, \lambda, \mu\right), 1 \leqslant i, j, k \leqslant n$, such that:

$$
\begin{aligned}
& \lambda u^{1}+\mu v^{1}=0, \\
& \lambda u^{2}+\mu v^{2}=q_{1}, \\
& \lambda u^{4}+\mu v^{4}=q_{3}, \\
& \lambda u^{i}+\mu v^{i}=0, \quad 5 \leqslant i \leqslant n, \\
& u^{1} q_{1}+u^{3} q_{3}=0, \\
& v^{1} q_{1}+v^{3} q_{3}=0, \\
& \lambda \sum_{i=1}^{4} u^{i} P_{i}(q)=1
\end{aligned}
$$

where the $P_{i}(q)$ are the $q_{i}$-coordinates of the vector field $\pi$, defined by formula (17) in the $p_{i}$-coordinates.

All these equations are independent, so that $\mathcal{M}$ is a submanifold of codimension $n+2$ in $\mathbb{R}^{3 n+2}$, and hence dimension $2 n$. 
Note that all points in $\mathcal{M}$ satisfy additional relations. From (42) and (43) we deduce that $u^{1} / v^{1}=u^{3} / v^{3}$, and equation (38) then implies that:

$$
\lambda u^{3}+\mu v^{3}=0 .
$$

Remembering the Walras law, we also find that Eq. (44) implies a similar relation for $\mu$ :

$$
\mu \sum_{i=1}^{4} v^{i} P_{i}=\left(\mu \sum_{i=1}^{4} v^{i} P_{i}+\lambda \sum_{i=1}^{4} u^{i} P_{i}\right)-\lambda \sum_{i=1}^{4} u^{i} P_{i}=1 .
$$

Consider the exterior differential system on $\mathcal{M}$ :

$$
\begin{aligned}
& \sum_{i=1}^{4} \mathrm{~d} u^{i} \wedge \mathrm{d} q_{i}=0, \\
& \sum_{i=1}^{4} \mathrm{~d} v^{j} \wedge \mathrm{d} q_{j}=0, \\
& \mathrm{~d} q_{1} \wedge \cdots \wedge \mathrm{d} q_{n} \neq 0,
\end{aligned}
$$

where the functions $P_{i}\left(q^{1}, \ldots, q^{n}\right), 1 \leqslant i \leqslant n$, are given.

Lemma 7. Any integral manifold of this system is the graph of a map:

$$
q \rightarrow\left(\frac{\partial u}{\partial q_{i}}, \frac{\partial v}{\partial q_{j}}, \lambda, \mu\right)
$$

where the functions $u, v, \lambda, \mu$ satisfy Eqs. (21)-(23) in the $p_{i}$-coordinates.

Proof. Condition (45) mean that the functions $u^{i}$ do not depend on the $q_{j}, 5 \leqslant j$, and that the cross-derivatives $\partial u^{i} / \partial q_{j}$ and $\partial u^{j} / \partial q_{i}$ are equal, for $1 \leqslant i, j \leqslant 4$. By the Poincaré lemma, there is a function $u\left(q_{1}, q_{2}, q_{3}, q_{4}\right)$ such that $u^{i}=\partial u / \partial q_{i}$. Similarly, condition (46) means that there is a function $v\left(q_{1}, q_{2}, q_{3}, q_{4}\right)$ such that $v^{i}=\partial v / \partial q_{i}$. These partial derivatives lie in $\mathcal{M}$ for all $q$. Going back to the $p_{i}$-coordinates, this means precisely that they satisfy all the equations (21)-(23).

We claim that the Cartan-Kähler theorem applies, so that there is an integral manifold going through any given integral element. To prove it, we will have to check successively that the system is closed, that there is an integral element through every point in $\mathcal{M}$, and that the Cartan test is satisfied.

It is obvious from the form of Eqs. (45) and (46) that the system is closed.

Let us now look for integral elements. Setting:

$$
\begin{aligned}
& d u^{i}=\sum_{j=1}^{n} U^{i j} \mathrm{~d} q_{j}, \\
& d v^{i}=\sum V^{i j} \mathrm{~d} q_{j}, \\
& d \lambda=\sum L^{j} \mathrm{~d} q_{j}, \\
& d \mu=\sum M^{j} \mathrm{~d} x_{j}
\end{aligned}
$$

and substituting in Eqs. (45) and (46) we get:

$$
\begin{aligned}
& U^{i j}-U^{j i}=0, \quad 1 \leqslant i, j \leqslant 4, i \neq j, \\
& V^{i j}-V^{j i}=0, \quad 1 \leqslant i, j \leqslant 4, i \neq j, \\
& U^{i j}=0, \quad 1 \leqslant i \leqslant 4 ; \quad 5 \leqslant j, \\
& V^{i j}=0, \quad 1 \leqslant i \leqslant 4 ; 5 \leqslant j .
\end{aligned}
$$


Note that the $U^{i j}$, the $V^{i j}$, the $L^{j}$ and the $M^{j}$ also have to satisfy the relations obtained by differentiating Eqs. (38)(44), which express that we are working in the Grassmannian bundle of $n$-planes in $T^{*} \mathcal{M}$. All these equations are linearly independent. Eqs. (48)-(51) number $12+4(n-4)+4(n-4)=8 n-20$, so that the set of integral elements has codimension $c=8 n-20$ in the Grassmannian.

Let us now perform Cartan's test. Write:

$$
\begin{aligned}
& \alpha^{i}=\mathrm{d} u^{i}-\sum_{i=1}^{4} U^{i j} \mathrm{~d} q_{j}, \\
& \beta^{i}=\mathrm{d} v^{i}-\sum_{i=1}^{4} V^{i j} \mathrm{~d} q_{j} .
\end{aligned}
$$

Note that, because of relations (48) to (51), we have

$$
\begin{aligned}
& \sum \alpha^{i} \wedge \mathrm{d} q_{i}=\sum \mathrm{d} u^{i} \wedge \mathrm{d} q_{i}=0, \\
& \sum \beta^{i} \wedge \mathrm{d} q_{i}=\sum \mathrm{d} v^{i} \wedge \mathrm{d} q_{i}=0 .
\end{aligned}
$$

We then apply the Cartan procedure, as described in [3]. We have:

$$
\begin{aligned}
& H_{0}=\{0\}, \\
& H_{1}=\operatorname{span}\left[\alpha^{1}, \beta^{1}\right], \\
& H_{2}=\operatorname{span}\left[\alpha^{1}, \beta^{1}, \alpha^{2}, \beta^{2}\right], \\
& H_{3}=\operatorname{span}\left[\alpha^{1}, \beta^{1}, \alpha^{2}, \beta^{2}, \alpha^{3}, \beta^{3}\right], \\
& H_{i}=\operatorname{span}\left[\alpha^{1}, \beta^{1}, \alpha^{2}, \beta^{2}, \alpha^{3}, \beta^{3}, \alpha^{4}, \beta^{4}\right] \quad \text { for } 4 \leqslant i
\end{aligned}
$$

and hence:

$$
\begin{aligned}
& c_{0}=0, \quad c_{1}=2, \quad c_{2}=4, \quad c_{3}=6, \quad c_{4}=\cdots=c_{n-1}=8, \\
& C=c_{0}+\cdots+c_{n-1}=0+2+4+6+8(n-4)=8 n-20
\end{aligned}
$$

which is exactly the codimension we found earlier: $C=c$. So the exterior differential system passes the Cartan test. By the Cartan-Kähler theorem, for every point $\left(q_{i}, u^{j}, v^{k}, \lambda, \mu\right), 1 \leqslant i, j, k \leqslant n$ in $\mathcal{M}$, and every integral element $\left(U^{i j}, V^{k \ell}, L^{r}, M^{s}\right)$ through that point, there is an integral manifold of the exterior differential system (45)-(47).

Of course, the conclusion is coordinate-independent. Let us revert to the original coordinates in $\mathbb{R}^{n}$, so that $\omega$ no longer has the special form $q_{1} \mathrm{~d} q_{2}+q_{3} \mathrm{~d} q_{4}$. The relations defining an integral element are then obtained directly by writing that they are tangent to holonomic sections of $\mathcal{M}$. A point in $\mathcal{M}$ is then specified by coordinates $\left(p_{i}, u^{j}, v^{k}, \lambda, \mu\right), 1 \leqslant i, j, k \leqslant n$ satisfying relations (27), (28) and (29). Introduce the notations:

$$
\xi=\left(u^{1}, \ldots, u^{n}\right), \quad \eta=\left(v^{1}, \ldots, v^{n}\right) .
$$

An integral element then is simply a set $(U, V, L, M)$, where $U$ and $V$ are symmetric $n \times n$ matrices, and $L$ and $M$ are $n$-vectors satisfying:

$$
\begin{aligned}
& \Omega=\lambda U+\mu V+L \xi^{\prime}+M \eta^{\prime}, \\
& \lambda U p=-\left(\xi^{\prime} p\right) L-\lambda \xi, \\
& \mu V p=-\left(\eta^{\prime} p\right) M-\mu \eta .
\end{aligned}
$$

Here $\Omega$ is the Jacobian matrix $\partial x^{i} / \partial p_{j}$. Note for future use that, by differentiating the relation $\sum x^{i}(p) p_{i}=2$ with respect to $p$, we get

$$
\sum_{i=1}^{n} \frac{\partial x^{i}}{\partial p_{j}} p_{i}+x^{j}=0,
$$


so that:

$$
\Omega p+\lambda \xi+\mu \eta=0 .
$$

The Cartan-Kähler theorem tells us that, given such an integral element at $(p, \xi, \eta, \lambda, \mu)$, there are functions $(u, v, \lambda, \mu)$ such that:

$$
\begin{aligned}
& D^{2} u(\bar{p})=U, \quad D^{2} v(\bar{p})=V, \\
& D u(\bar{p})=\xi, \quad D v(\bar{p})=\eta .
\end{aligned}
$$

\subsection{Finding convex solutions}

Given relations (56) and (57), all we have to prove is that there is an integral element $(U, V, L, M)$ at $\bar{p}$ with $U$ and $V$ positive definite. Then $D^{2} u(\bar{p})$ and $D^{2} v(\bar{p})$ will be positive definite, and so $u$ and $v$ will be strictly convex in a neighbourhood of $\bar{p}$.

To do this, we shall use a lemma of Pierre-Louis Lions [11]:

Lemma 8. Given a symmetric, positive definite matrix $Q$, a vector $p$ and two vectors $q_{1}$ and $q_{2}$ satisfying $q_{1}+q_{2}=$ $Q p$, a necessary and sufficient condition for the existence of two symmetric, positive definite matrices $Q_{1}$ and $Q_{2}$ such that:

$$
\begin{aligned}
& Q=Q_{1}+Q_{2}, \\
& Q_{1} p=q_{1}, \\
& Q_{2} p=q_{2}
\end{aligned}
$$

is that:

$$
\begin{aligned}
& \left(p, q_{1}\right)>0, \\
& \left(p, q_{2}\right)>0, \\
& \left(q_{1}, Q^{-1} q_{2}\right)>0 .
\end{aligned}
$$

Proof. Let us set $x=Q^{1 / 2} p, y=Q^{-1 / 2} q_{1}$ and $z=Q^{-1 / 2} q_{2}$. Set also

$$
M=Q^{-1 / 2} Q_{1} Q^{-1 / 2} \text { and } N=Q^{-1 / 2} Q_{2} Q^{-1 / 2} .
$$

We have:

$$
\begin{aligned}
& M+N=I, \\
& M x=y, \\
& N x=z
\end{aligned}
$$

and $y+z=x$. So it is enough to prove the lemma for the particular case where $Q=I$. Let us do it.

Using the three equations, we find easily:

$$
\begin{aligned}
& (x, y)=(x, M x)>0, \\
& (x, z)=(x, N x)>0 .
\end{aligned}
$$

Both $M$ and $I-M$ are positive definite. This means that $0<M<I$ in the sense of symmetric matrices, so that $M^{2}<M$. It follows that:

$$
(y, z)=(M x, N x)=(M x,(I-M) x)=(x, M x)-\left(x, M^{2} x\right)>0 .
$$

So the conditions (58), (59), and (60) are necessary.

Let us now prove that they are sufficient. Given three vectors $x, y, z$ satisfying (64), (65), and (66) we seek a symmetric matrix $M$ such that $M x=y$ and $0<M<1$. Indeed, we then set $N=I-M$, which is still symmetric and positive definite, and we have $N x=x-M x=x-z=y$, as desired. 
It is enough to solve the problem in the plane spanned by $x$ and $y$. Without loss of generality, we assume that $\|x\|=1$. Take an orthonormal basis $\left(\vec{e}_{1}, \vec{e}_{2}\right)$ in that plane with $x=(1,0)$ and $y=\left(y_{1}, y_{2}\right)$. Eqs. (64), (65) and (66) yield $0<y_{1}<1$ and $y_{1}-\|y\|^{2}>0$.

The matrix we are looking for is:

$$
M=\left(\begin{array}{cc}
y_{1} & y_{2} \\
y_{2} & c
\end{array}\right)
$$

where we will adjust the constant $c$. Since $(x, y)$ and $(x, z)$ are positive, we have $0<y_{1}<1$.

Clearly $M x=y$. We will adjust the constant $c$ to have $0<M<I$. This requires that the determinant and the trace of $M$ and $(I-M)$ are positive.

Writing that both determinants are positive, we get:

$$
\frac{y_{2}^{2}}{y_{1}}<c<1-\frac{y_{2}^{2}}{1-y_{1}} \text {. }
$$

Since $y_{1}>\|y\|^{2}$, the left-hand side is smaller than the right-hand side, so we can find some $c$ in that interval. Since $0<y_{1}<1$, we must have $0<c<1$.

Writing that both traces are positive, we get:

$$
-y_{1}<c<2-y_{1} \text {. }
$$

Since $0<y_{1}<1$, this inequality follows from $0<c<1$. So any $c$ that satisfies the first inequality satisfies the second.

We now return to our main argument. By assumption, the GS $(k)$ condition is satisfied, so that there is a symmetric, positive definite $n \times n$ matrix $Q$ and $n$-vectors $L$ and $M$ such that:

$$
\Omega=Q+L \xi^{\prime}+M \eta^{\prime}
$$

Lemma 9. Denote by $L_{0}$ and $M_{0}$ the projections of $L$ and $M$ on $[\xi, \eta]^{\perp}$ and by $Q_{0}$ the restriction of $Q$ to $[\xi, \eta]^{\perp}$. Without loss of generality, it can be assumed that

$$
\left(\xi^{\prime} p\right)\left(\eta^{\prime} p\right) L_{0}^{\prime} Q_{0}^{-1} M_{0}>0 .
$$

Proof. Take any numbers $\alpha, \beta, \gamma$ and $\delta$ such that $\beta \gamma-\alpha \delta=1$. Rewrite $\Omega$ as follows:

$$
\Omega=Q+\bar{L} \bar{\xi}^{\prime}+\bar{M} \bar{\eta}^{\prime}
$$

with:

$$
\begin{array}{ll}
\bar{L}=\alpha L+\beta M, & \bar{M}=\gamma L+\delta M, \\
\bar{\xi}=-\delta \xi+\gamma \eta, & \bar{\eta}=\beta \xi-\alpha \eta .
\end{array}
$$

We then have:

$$
\begin{aligned}
& \left(\bar{\xi}^{\prime} p\right)\left(\bar{\eta}^{\prime} p\right) \bar{L}_{0}^{\prime} Q_{0}^{-1} \bar{M}_{0}=\left[-\delta\left(\xi^{\prime} p\right)+\gamma\left(\eta^{\prime} p\right)\right]\left[\beta\left(\xi^{\prime} p\right)-\alpha\left(\eta^{\prime} p\right)\right]\left(\alpha L_{0}^{\prime}+\beta M_{0}^{\prime}\right) Q_{0}^{-1}\left(\gamma L_{0}+\delta M_{0}\right) \\
& \quad=\left[-\delta\left(\xi^{\prime} p\right)+\gamma\left(\eta^{\prime} p\right)\right]\left[\beta\left(\xi^{\prime} p\right)-\alpha\left(\eta^{\prime} p\right)\right]\left[\alpha \gamma L_{0}^{\prime} Q_{0}^{-1} L_{0}+(\alpha \delta+\beta \gamma) L_{0}^{\prime} Q_{0}^{-1} M_{0}+\beta \delta M_{0}^{\prime} Q_{0}^{-1} M_{0}\right] .
\end{aligned}
$$

Set the value of $\delta$ to $\bar{\delta}=\left(\eta^{\prime} p\right)$. The first term on the right-hand side then changes sign for $\gamma=\bar{\gamma}=\left(\xi^{\prime} p\right)$. We will find values $\bar{\alpha}$ and $\bar{\beta}$, with $\bar{\gamma} \bar{\beta}-\bar{\delta} \bar{\alpha}=1$ such that the two other terms are non-zero for $(\bar{\alpha}, \bar{\beta}, \bar{\gamma}, \bar{\delta})$. It then follows that the right hand side has two opposite signs for $(\bar{\alpha}, \bar{\beta}, \bar{\gamma}+\varepsilon, \bar{\delta})$ and for $(\bar{\alpha}, \bar{\beta}, \bar{\gamma}-\varepsilon, \bar{\delta})$, when $\varepsilon>0$ is small enough, and the result obtains.

Rewrite Eq. (67) as follows:

$$
\Omega=Q+\alpha \xi \xi^{\prime}+\beta \eta \eta^{\prime}+(L-\alpha \xi) \xi^{\prime}+(M-\beta \eta) \eta^{\prime}
$$


with $\alpha$ and $\beta \geqslant 0$, so that the matrix $Q+\alpha \xi \xi^{\prime}+\beta \eta \eta^{\prime}$ is positive definite. Let us apply the lemma of Pierre-Louis Lions to find positive definite matrices $Q_{1}$ and $Q_{2}$ such that:

$$
\begin{aligned}
& Q_{1}+Q_{2}=Q+\alpha \xi \xi^{\prime}+\beta \eta \eta^{\prime}, \\
& Q_{1} p=-\left(\xi^{\prime} p\right)(L-\alpha \xi)-\lambda \xi, \\
& Q_{2} p=-\left(\eta^{\prime} p\right)(M-\beta \eta)-\mu \eta .
\end{aligned}
$$

We first check that $Q_{1} p$ and $Q_{2} p$ add up to $Q p+\alpha \xi\left(\xi^{\prime} p\right)+\beta \eta\left(\eta^{\prime} p\right)$. Using (68), and (52), we have:

$$
\begin{aligned}
& Q_{1} p+Q_{2} p=-\left(\xi^{\prime} p\right)(L-\alpha \xi)-\left(\eta^{\prime} p\right)(M-\beta \eta)-\lambda \xi-\mu \eta, \\
& Q p+\alpha \xi\left(\xi^{\prime} p\right)+\beta \eta\left(\eta^{\prime} p\right)=\Omega p-(L-\alpha \xi)\left(\xi^{\prime} p\right)-(M-\beta \eta)\left(\eta^{\prime} p\right) .
\end{aligned}
$$

Subtracting, we get:

$$
Q p-Q_{1} p-Q_{2} p=\Omega p+\lambda \xi+\mu \eta
$$

and the right-hand side vanishes by relation (55).

The first condition holds, and we proceed to the others. Conditions (58) and (59) become:

$$
\begin{aligned}
& -\left(\xi^{\prime} p\right)\left[\left(p^{\prime} L\right)-\alpha\left(\xi^{\prime} p\right)-\lambda\right]>0, \\
& -\left(\eta^{\prime} p\right)\left[\left(p^{\prime} M\right)-\beta\left(\eta^{\prime} p\right)-\mu\right]>0
\end{aligned}
$$

both of which hold true for $\alpha>0$ and $\beta>0$ large enough.

When $\alpha, \beta \rightarrow \infty$, we have, for any $\xi$ and $\eta$ in $\mathbb{R}^{n}$ :

$$
\left(\xi,\left[Q+\alpha \xi \xi^{\prime}+\beta \eta \eta^{\prime}\right]^{-1} \eta\right) \rightarrow\left(\xi_{0}, Q_{0}^{-1} \eta_{0}\right)
$$

where the subscript 0 denotes the projection on $[\xi, \eta]^{\perp}$. Applying this with $\xi=-\left(\xi^{\prime} p\right)(L-\alpha \xi)-\lambda \xi$ and $\eta=$ $-\left(\eta^{\prime} p\right)(M-\beta \eta)-\mu \eta$, we find:

$$
\left(\xi_{0}, Q_{0}^{-1} \eta_{0}\right)=\left(\xi^{\prime} p\right)\left(\eta^{\prime} p\right) L_{0}^{\prime} Q_{0}^{-1} M_{0}
$$

and the right-hand side is positive by the lemma.

The proof is thus happily concluded in the case $k=2$. In the case $k>2$, the same argument goes through, except for the last Lemma, which relies on the fact that $M$ and $(I-M)$ commute. One must then use another characterisation, which is due to Inchakov [10]. Without loss of generality, assume $Q=I$, and consider the quadratic form:

$$
(C z, z)=\sum \frac{\left(x_{n}, z\right)^{2}}{\left(x_{n}, y\right)}-(z, z)
$$

Lemma 10. Assume $\left(x_{n}, y\right) \neq 0$ for all $n$. Then a necessary and sufficient condition for the existence of positive definite matrices $M_{n}$ such that $M_{n} y=x_{n}$ for all $n$ and $\sum M_{n}=I$ is that $\sum x_{n}=y,\left(x_{n}, y\right)>0$ for all $n$, and $(C z, z)<0$ for all $z$ not collinear with $y$.

A proof of Inchakov's lemma is provided in [7].

\section{Conclusion}

We would like to conclude with a remark of some economic interest. It has been shown by Browning and Chiappori [2] that condition GS( $k)$ is necessary in the case of household demand - that is, when there are public goods in the economy and externalities between consumers. In other words, if $x(p)$ is the demand function of a household with $k<n$ consumers, then it must satisfy $\mathrm{GS}(k)$. But it follows from our result that the same $x(p)$ also is the demand function of an exchange economy, where there are only private goods and no externalities. In other words, households and exchange economies cannot be distinguished by looking at the demand functions only. 


\section{References}

[1] G. Antonelli, Sulla teoria matematica della economia politica, Pisa, 1886; Translated in: J. Chipman, L. Hurwicz, M. Richter, H. Sonnenschein, et al. (Eds.), Preferences, Utility and Demand, Harcourt Brace Jovanovich, 1971.

[2] W. Browning, P.A. Chiappori, Efficient intra-household allocation: a general characterization and empirical tests, Econometrica 66 (6) (1998) $1241-1278$.

[3] R.L. Bryant, S.S. Chern, R.B. Gardner, H.L. Goldschmidt, P.A. Griffiths, Exterior Differential Systems, Springer-Verlag, New York, 1991.

[4] P.A. Chiappori, I. Ekeland, Aggregation and market demand: an exterior differential calculus viewpoint, Econometrica 67 (1999) $1435-1458$.

[5] P.A. Chiappori, I. Ekeland, Individual excess demands, J. Math. Economics, in press.

[6] W.E. Diewert, Generalized Slutsky conditions for aggregate consumer demand functions, J. Economic Theory 15 (1977) 353-362.

[7] I. Ekeland, Some applications of the Cartan-Kähler theorem to economic theory, in: Erich Kähler, Mathematische Werke, de Gruyter, Berlin, 2003, pp. 767-777.

[8] I. Ekeland, L. Nirenberg, A convex Darboux theorem, Methods Appl. Math. 9 (2002) 329-344.

[9] J. Geanakoplos, H. Polemarchakis, On the disaggregation of excess demand functions, Econometrica (1980) 315-333.

[10] Inchakov, Personal communication, 2000.

[11] P.L. Lions, Personal communication, Université Paris-Dauphine, 1998.

[12] A. Mas-Colell, M. Whinston, J. Green, Microeconomic Theory, Oxford University Press, 1995.

[13] W. Shafer, H. Sonnenschein, Market demand and excess demand functions, in: K. Arrow, M. Intriligator (Eds.), Handbook of Mathematical Economics, vol. 2, North-Holland, 1982 (Chapter 14).

[14] E. Slutsky, Sulla teoria del bilancio del consumatore, Giornale degli Economisti e Rivista di Statistica 3 (15) (1915) 1-26; English translation in: G. Stigler, K. Boulding (Eds.), Readings in Price Theory, Richard D. Irwin, 1952. 